\title{
Studies on the Positional Specificity of Lipase from Mucor miehei during Interesterification Reactions of Cod Liver Oil with $n-3$ Polyunsaturated Fatty Acid and Ethyl Ester Concentrates
}

\author{
Gudmundur G. Haraldsson and Örn Almarsson
}

Science Institute, University of Iceland, Dunhaga 3, 107 Reykjavik, Iceland

\begin{abstract}
Haraldsson, G. G. and Almarsson, Ö., 1991. Studies on the Positional Specificity of Lipase from Mucor miehei during Interesterification Reactions of Cod Liver Oil with n-3 Polyunsaturated Fatty Acid and Ethyl Ester Concentrates. - Acta Chem. Scand. 45: 723-730.

Lipozyme, a lipase preparation from Mucor miehei, is 1,3 specific in the interesterification of cod liver oil and fatty acid and ethyl ester concentrates of $n-3$ polyunsaturated fatty acids in organic media. Randomization of the fatty acid distribution in the mid-position of the glycerol moiety of triglycerides in cod liver oil occurs as a result of slow acyl migration processes. Acyl migration seems to be rate-determining in the attainment of a final equilibrium of fatty acid composition in triglycerides. This is especially apparent when fatty acid concentrates are used.
\end{abstract}

Lipases that exhibit activity towards glycerol esters are commonly specific for different sites on the glycerol backbone. ${ }^{1.2}$ This positional specificity is usually restricted to the outermost ester functions, i.e. positions 1 and 3 on a triglyceride (Fig. 1), and thus a lipase is 1,3 specific. More generally, 1,3 specific lipases are active on esters of primary alcohol, but very few lipases are enantioselective towards naturally occurring triglycerides and no reports have been made on 2-specific lipases.

The natural working environment for a lipase is an emulsion of substrate where the enzymes show hydrolytic activity at the interface between water and either a waterimmiscible substrate or substrate dissolved in an organic solvent. ${ }^{3}$ In the last decade work has commenced on the use of lipases and other enzymes in organic media. ${ }^{4,5}$ Water is eliminated, apart from essential water needed to maintain structural integrity of the enzyme molecules. As a consequence, lipases will catalyze the reverse reaction of hydrolysis, ester synthesis, as well as transesterification, alcoholysis and acidolysis, among others.

Our recently reported application of 1,3 specific lipase in interesterification reactions to produce $n-3$ (sometimes called omega-3) fatty acid enriched triglycerides ${ }^{6}$ makes use of the catalytic properties of lipase in a microaqueous environment. Ester interchange between $n-3$ polyunsaturated free fatty acid and ethyl ester concentrates with cod liver oil predominated over hydrolysis of the fat and triglycerides highly enriched in $n-3$ fatty acids were obtained. Attainment of equilibrium was rather slow, taking more than $24 \mathrm{~h}$.

An interesting question arose as to whether the mid-

${ }^{*}$ To whom correspondence should be addressed. position in the triglyceride was involved in the interesterification processes. Did the lipase strictly adhere to its regiospecificity, leaving the mid-position untouched during the interesterification processes? Or was the mid-position participating in an equilibrium leading to a random distribution of the fatty acids, presumably involving acyl migration promoted by the lipase? As has been summarized very recently by Bloomer ${ }^{7}$ in an excellent review of the acyl migration, such intramolecular processes are affected by various factors such as acidic or basic conditions, heat, ion-exchange supports for immobilized enzyme and the size of the acyl groups.

At the beginning it was not easy to deduce the role of the mid-position during the interesterification processes, because the mid-position in cod liver oil is known to be considerably more enriched with the $n-3$ polyunsaturated fatty acids. ${ }^{8}$ Several pieces of information appeared to indicate that the mid-position might not participate. In the present study, methods of analysis of the mid-position of triglycerides were employed to investigate the rate of changes in position 2 compared with positions 1 and 3 on the glycerol moiety during interesterification on cod liver oil catalyzed by a 1,3 specific lipase. Both transesterification with ethyl ester concentrates of $n-3$ (PUFEE) and acidolysis with $n-3$ concentrates of free fatty acids (PUFA) were studied. The incorporation of icosapentaenoic acid and docosahexaenoic acid as well as the expulsion of palmitic acid from the mid-position was used to monitor the course of events at the mid-position during the interesterification processes. In this respect palmitic acid was found to be very useful since the mid-position in the cod liver oil triglycerides is considerably more enriched with that acid compared with the end-positions, and the concentrates 


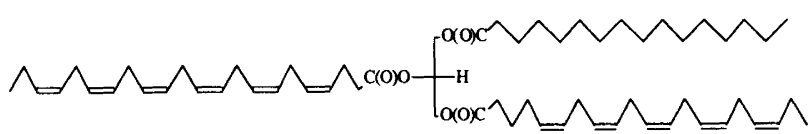

Fig. 1. A triglyceride of specific stereochemical structure: sn-1-palmitoyl-2-docosahexaenoyl-3-icosapentaenoyl glycerol (1-PA-2-DHA-3-EPA glycerol).

were virtually free of palmitic acid. Our results indicate that when equilibrium is attained, random distribution is indeed established.

\section{Materials and methods}

Materials. Medicinal cod liver oil was obtained from Lysi hf., Reykjavik and ethyl ester concentrates of $n-3$ fatty acids were prepared by successive urea inclusion and molecular distillation. ${ }^{9}$ Free fatty acid concentrates of essentially identical composition were obtained by alkaline hydrolysis of the ethyl esters. The cod liver oil contained 9.4, 9.2 and $10.7 \%$ of $(Z)-5,8,11,14,17$-icosapentaenoic acid (EPA), (Z)-4,7,10,13,16,19-docosahexaenoic acid (DHA) and palmitic acid (PA) as glyceryl esters, respectively. The concentrates contained $39.4 \%$ and $35.2 \%$ of EPA and DHA, respectively, with no detectable amounts of PA. The average molecular weights of all the substrates involved were calculated using weight percentages of methyl esters from GC analysis, taking into account the weight of the methyl unit. Lipozyme was supplied by Novo Nordisk, Bagsværd, Denmark and contained approximately $10 \%$ essential water by weight. Methylmagnesium bromide in ethyl ether $(3.0 \mathrm{M})$ was purchased from Aldrich, and was standardized prior to use by the double titration method of Gilman and Cartledge. ${ }^{10}$ Whatman 150A LK5 silica gel TLC plates were used after impregnation with a solution of boric acid according to the method of Fine and Specher ${ }^{11}$ and activation at $110^{\circ} \mathrm{C}$. Elution was performed with mixtures of analytical grade hexane and ethyl ether from Merck AG, Darmstadt, Germany. Ethyl ether was dried and distilled from sodium in the presence of benzophenone under an inert atmosphere immediately before use for Grignard reactions, but otherwise solvents were used without refinement. Rhodamine $6 \mathrm{G}$, magnesium sulfate, sodium hydroxide, calcium chloride and sodium hydrogencarbonate were obtained from Merck. Sodium hydroxide in methanol $(0.5 \mathrm{M})$ was prepared from analytical grade methanol from Merck. Boron trifluoride in methanol (14\%), neutral alumina, porcine pancreatic lipase (Type II, crude), sodium taurocholate $(98 \%)$ and Trizma base ${ }^{\circledR}$ $(99.9 \%)$ were purchased from Sigma.

Alkaline hydrolysis of ethyl ester concentrates. Ethyl esters $(50 \mathrm{~g}, 0.15 \mathrm{~mol})$ were added to a solution of sodium hydroxide $(15 \mathrm{~g}, 0.38 \mathrm{~mol})$ and ethylenediaminetetraacetic acid, disodiumsalt dihydrate $\left(\mathrm{Na}_{2}\right.$ EDTA $\left.\cdot \mathrm{H}_{2} \mathrm{O} ; 0.15 \mathrm{~g}\right)$ in a mix- ture of ethanol $(96 \%, 50 \mathrm{ml})$ and distilled water $(50 \mathrm{ml})$. The resulting mixture was heated at $60-65^{\circ} \mathrm{C}$ and magnetically stirred under nitrogen atmosphere for $30 \mathrm{~min}$. The homogeneous mixture was allowed to cool and acidified with concentrated hydrochloric acid (approximately $50 \mathrm{ml}$ ) until pH 1 was reached (indicator paper). The organic phase was separated and the aqueous phase extracted with hexane $(40 \mathrm{ml})$. The combined organic phases were washed with water $(2 \times 50 \mathrm{ml})$ and brine $(50 \mathrm{ml})$. The organic phase was dried over anhydrous magnesium sulfate and the organic solvent was removed in vacuo. The final traces of solvent were removed in vacuo, with the aid of a high-vacuum pump, to afford the free acids as a yellow oil in almost quantitative yield.

Interesterifications of cod liver oil with $\mathrm{n}-3$ polyunsaturated concentrates. Lipozyme $(6.0 \mathrm{~g})$ was added to a gently stirred mixture of ethyl esters $(45.0 \mathrm{~g}$; mol. wt. 342.4, 0.131 $\mathrm{mol})$ or free fatty acids $(45.0 \mathrm{~g}$; mol. wt. $314.4,0.143 \mathrm{~mol})$ and medicinal cod liver oil $(15.0 \mathrm{~g}$, mol. wt. 894.0, 0.0168 $\mathrm{mol}$ ) in a $250 \mathrm{ml}$ two-necked round-bottom flask. The mixture was kept at $60-65^{\circ} \mathrm{C}$ under nitrogen and samples were withdrawn over a period of $72 \mathrm{~h}$ and passed through a cotton wool plug. The lipase-free aliquots were subjected to purification and Grignard reactions.

Purification of triglycerides from transesterification. A lipase-free reaction mixture sample (3-4 g) was dissolved in $10 \%$ ethyl ether in petroleum ether $(4 \mathrm{ml})$ and the solution was injected into a Waters PrepLC ${ }^{\mathrm{TM}}$ System 500A instrument. The PrepPak ${ }^{\circledR} 500 /$ Silica Cartridge column from Millipore was eluted with $10 \%$ ethyl ether in petroleum ether. Each run afforded two peaks according to the index of refraction detector, the first appearing after approximately one column volume and consisting of pure residual ethyl esters and the second peak eluting after roughly two column volumes and containing around $99 \%$ pure triglycerides, as established by iatroscan analysis. ${ }^{12}$ The solvent was removed in vacuo and the triglyceride fraction was used directly in a Grignard reaction.

Purification of triglycerides from acidolysis. A lipase-free reaction mixture sample $(3-4 \mathrm{~g})$ was placed in a separatory funnel in hexane $(50 \mathrm{ml})$ along with $0.25 \mathrm{M}$ sodium hydroxide in $1: 1(\mathrm{v} / \mathrm{v})$ water-ethanol solution $(50 \mathrm{ml})$. The mixture was shaken and allowed to separate and the aqueous layer was drawn off and extracted twice with base $(2 \times 30 \mathrm{ml})$. The combined basic aliquots were extracted with hexane $(30 \mathrm{ml})$ and the combined extracts were washed once more with base solution $(25 \mathrm{ml})$, water $(30 \mathrm{ml})$ and brine $(30 \mathrm{ml})$. The organic layer was dried over anhydrous magnesium sulfate and evaporated in vacuo. The resulting fat was dissolved in dry ethyl ether $(50 \mathrm{ml})$ and run through a column containing neutral alumina. The column was further rinsed with diethyl ether $(50 \mathrm{ml})$, the two ether aliquots combined, the ether removed in vacuo and the resulting glyceride fraction used in a Grignard reaction. 
Grignard reaction of triglycerides from interesterifications. The reaction was run in flame-dried glassware and under nitrogen according to the method of Brockerhoff. ${ }^{13}$ To a solution of triglyceride $(1.0 \mathrm{~g}$, ca. $1.1 \mathrm{mmol})$ in dry ethyl ether $(50 \mathrm{ml})$ at ambient temperature was added $3.0 \mathrm{M}$ methylmagnesium bromide $(2.0 \mathrm{ml}, 6.0 \mathrm{mmol})$ via syringe. The solution was stirred under nitrogen for $30 \mathrm{~s}$ and quenched by addition of acetic acid $(1 \mathrm{ml})$ and, $30 \mathrm{~s}$ later, water $(10 \mathrm{ml})$. The resulting mixture was stirred for another $2 \mathrm{~min}$ and the ether layer separated, washed with water (10 $\mathrm{ml}), 10 \%$ aqueous sodium hydrogencarbonate $(10 \mathrm{ml})$ and water $(10 \mathrm{ml})$. The organic phase was dried over anhydrous magnesium sulfate and the solvent removed in vacuo to afford the product mixture as an oil. The mixture was separated by preparative TLC, using $8 \%$ ethyl ether in petroleum ether as the eluent, followed by a second elution with $40 \%$ ethyl ether in petroleum ether. Visualization was achieved with a $0.12 \%$ Rhodamine $6 \mathrm{G}$ solution in water followed by inspection under short-wavelength $U V$. 1,3-diglycerides had an $R_{\mathrm{f}}$ of 0.45 , whereas $1,2(2,3)$ diglycerides were slower, with an $R_{\mathrm{f}}$ of 0.36 . Glyceride bands were scraped off and methylated (vide infra). Both 1,2(2,3)- and 1,3-diglycerides were analyzed by capillary GC and used to calculate the composition in the midposition. 1,3-diglycerides were used as a measure of the average composition in the end-positions.

Pancreatic lipase catalyzed hydrolysis of triglycerides from interesterifications. The reaction was run according to the method of Mattson and Volpenhein, ${ }^{14}$ typically using reaction times of 4 or $5 \mathrm{~min}$. To a suspension of triglycerides $(500 \mathrm{mg})$ in aqueous $0.2 \%(\mathrm{w} / \mathrm{v})$ sodium taurocholate $(3.7$ $\mathrm{ml})$ and $2.0 \mathrm{M}$ calcium chloride $(1.5 \mathrm{ml})$, was added the supernatant of a solution of porcine pancreatic lipase $(250$ $\mathrm{mg}$ ) in $0.1 \mathrm{M}$ Tris buffer $(15 \mathrm{ml})$, at $\mathrm{pH} 8.0$ at $40^{\circ} \mathrm{C}$, prepared from Trizma base ${ }^{\circledR}$ by titration with $6 \mathrm{M}$ hydrochloric acid. The resulting emulsion was shaken for $30 \mathrm{~s}$, then left at $40^{\circ} \mathrm{C}$ for the remainder of the reaction time. Quenching was effected by addition of $0.05 \mathrm{M}$ hydrochloric acid in 1:1 ethanol-water $(20 \mathrm{ml})$. Extraction was performed with $1: 1$ hexane-ethyl ether $(3 \times 40 \mathrm{ml})$ and the organic phase was washed with $1: 1$ ethanol-water $(2 \times 40$ $\mathrm{ml})$ and brine $(40 \mathrm{ml})$. The organic phase was dried over anhydrous magnesium sulfate and solvent removed in $v a$ cuo. The progress of hydrolysis was assessed by titration with base after isolation of the product mixture by extraction. 2-Monoglycerides, representative for the composition of the mid-position in the triglycerides, were isolated by preparative TLC eluting with $60: 40: 3$ petroleum etherethyl ether-acetic acid $\left(R_{\mathrm{f}}=0.08\right)$. The band was scraped off, methylated (vide infra) and analyzed by GC.

Gas chromatography. Gas chromatography was performed on a Perkin-Elmer 8140 Gas Chromatograph using a 30 meter capillary column, DB-22530N $0.25 \mu \mathrm{m}$. Hydrogen was used as the carrier gas at a flow rate of $1.6 \mathrm{ml} \mathrm{min}^{-1}$. Detection was with FID and areal quantitation was made with an auxiliary automatic integrator. A temperature of $180^{\circ} \mathrm{C}$ was used at the beginning, rising $3^{\circ} \mathrm{min}^{-1}$ to a final temperature of $210^{\circ} \mathrm{C}$, which was maintained for $12 \mathrm{~min}$. Injector and detector were kept at $265^{\circ} \mathrm{C}$.

Methyl esters were routinely prepared from triglycerides using a modified method, based largely on the procedure of Einig and Ackman. ${ }^{15}$ Four drops of oil were placed in a 20 $\mathrm{ml}$ screw cap tube with $0.5 \mathrm{M}$ sodium hydroxide in methanol $(2.0 \mathrm{ml})$. Nitrogen was blown over, the cap was placed tightly on and the tube shaken vigorously, placed in a steam bath at $65^{\circ} \mathrm{C}$ for $5-10 \mathrm{~min}$, until the droplets of fat had dissolved. The tube was then cooled briefly, opened carefully and $14 \%$ boron trifluoride in methanol $(2.5 \mathrm{ml})$ was added. The cap was immediately replaced and the tightly closed vessel heated on the steam bath for another $20 \mathrm{~min}$. When the tube had been cooled to room temperature, hexane $(2 \mathrm{ml})$ and water $(3 \mathrm{ml})$ were added and the tube was shaken well. The clear hexane extract was removed with a pipette and fresh hexane $(1 \mathrm{ml})$ was added, the tube shaken and the hexane again removed and combined with the earlier fraction. The resulting solution $(2 \mu \mathrm{l})$ was analyzed by GC using a split ratio of 1:150.

Slight modifications are required when other forms of fat are dealt with. When free fatty acids were methylated only the latter part of the above procedure applied. Four drops of the oil were placed in a $20 \mathrm{ml}$ screw cap tube, boron trifluoride-methanol solution $(2.0 \mathrm{ml})$ was added and the procedure was carried on from there. Glyceride bands on silica gel were scraped off and placed in a screw-cap tube and the procedure for triglycerides was followed until the extraction steps were reached. Hexane $(2 \times 3 \mathrm{ml})$ was used for extraction, combined volumes placed in a dry $10 \mathrm{ml}$ test tube and the solvent evaporated to dryness under a stream of nitrogen. The residual methyl esters were redissolved in a suitable volume so that the solution was approximately 25 $\mathrm{mg} \mathrm{ml} \mathrm{m}^{-1}$. Ethyl esters were usually analyzed directly as such, but the procedure for triglycerides can be followed to analyze them as methyl esters if desired.

\section{Results and discussion}

In the interesterification studies demonstrated in this paper, ethyl ester and free fatty acid concentrates of identical composition, $39.4 \%$ EPA and $35.2 \%$ DHA, were used for the transesterification and acidolysis processes with cod liver oil, respectively. In all experiments the triglyceride to concentrate ratio was kept at 1:3, based on weight, which is equivalent to an approximately 2.6 and 2.8 fold excess, respectively, of ethyl esters and free fatty acids, based on the number of acyl equivalents in the triglyceride moiety of cod liver oil.

As mentioned earlier, the point at issue here is the role of the mid-position of the triglycerides in the interesterification processes described. Eqns. (1) and (2) below have been deduced and used to calculate percentage composition of any fatty acid participating in such a process at an 
equilibrium: eqn, (1) for the mid-position freely participating and the fatty acids equally and randomly distributed among all positions of the triglyceride, and eqn. (2) for the mid-position not participating, leaving its composition unchanged. $X_{\mathrm{TG}}$ represents any participating fatty acid in the

$\% X_{\mathrm{TG}}=\frac{a}{a+b} \% X_{\mathrm{CLO}}+\frac{b}{a+b} \% X_{\mathrm{PUFA}}$

$\% X_{\mathrm{TG}}=\frac{1}{3} \% X_{\mathrm{CLO}_{-2}}+$

$\frac{2}{3}\left(\frac{\frac{2}{3} a}{\frac{2}{3} a+b} \% X_{\mathrm{CLO}_{-1,3}}+\frac{b}{\frac{2}{3} a+b} \% X_{\mathrm{PUFA}}\right)$

final product at equilibrium, $X_{\mathrm{CLO}}$ the composition of that fatty acid in the initial cod liver oil substrate, $X_{\text {PUFA }}$ the composition in the concentrate, either free fatty acid or ethyl ester, $X_{\mathrm{CLO}_{-2}}$ the composition in the mid-position of cod liver oil and $X_{\mathrm{CLO}_{-1.3}}$ represents the composition of the acid in the end-positions of cod liver oil. The coefficients $a$ and $b$ represent the number of acyl equivalents in the cod liver oil and the concentrate, respectively.

Incorporation of $\mathrm{n}-3$ fatty acids into triglycerides during transesterification. The triglycerides from HPLC purification were subjected to fatty acid analysis as methyl esters on GC. The rate of uptake of $n-3$ fatty acids into di- and tri-glycerides as well as the rate of exclusion of PA were monitored as a function of time, the results being shown in Fig. 2. EPA is incorporated and palmitic acid disappears very rapidly, while DHA is taken up at a slower rate. The equilibrium concentrations of these acids were found to be $33.0,2.8$ and $28.7 \%$, respectively, which are close to the figures expected at random distribution according to eqn. (1) (see Table 1).

The strange behaviour of EPA in the first few hours is presumably a result of fatty acid selectivity of lipozyme for this acid over DHA, which was attributed to the fact that the double bond closest to the carboxy group is located one

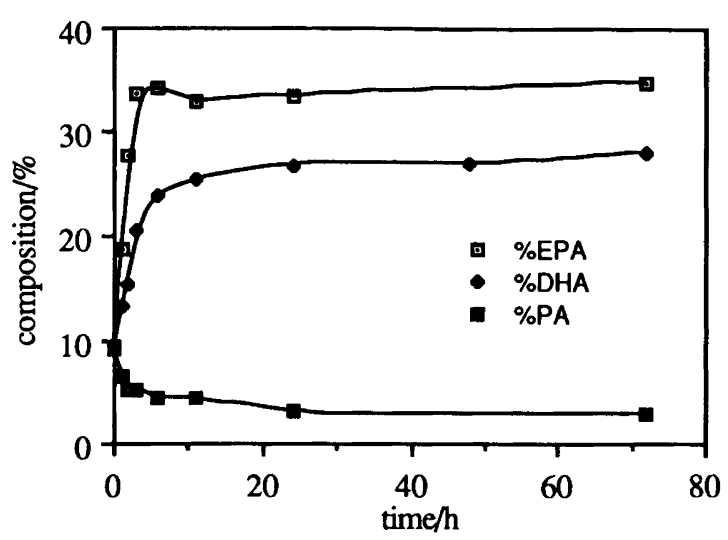

Fig. 2. Percentages of EPA, DHA and PA in triglycerides during transesterification.

carbon-carbon bond closer in the DHA molecule. ${ }^{16}$ Momentarily, the composition of EPA in the glycerides exceeds the statistical equilibrium level, which was reached in $10 \mathrm{~h}$.

Incorporation of $\mathrm{n}-3$ fatty acids into triglycerides during acidolysis. Acidolysis of cod liver oil was conducted in the same manner as transesterification, only using free acids instead of ethyl esters. The glyceride fractions from extraction with alkaline water-alcohol solution and further purification on a column containing neutral alumina were analyzed, after methylation, by GC.

Uptake of fatty acids is apparently much slower in this reaction compared with transesterification, DHA showing the greatest difference in rate (cf. Fig. 3 with Fig. 2).

The equilibrium concentrations of EPA, DHA and PA were found to be $33.5,26.1$ and $3.2 \%$, respectively, the DHA percentage being significantly lower than after transesterification and \% PA being slightly higher. In contrast, EPA gave a similar result in both systems, although somewhat slower in the acidolysis case.

Table 1. Comparison of fatty acid composition in glyceride fractions according to measurements and calculations.

\begin{tabular}{|c|c|c|c|c|c|c|}
\hline \multirow{4}{*}{$\begin{array}{l}\text { Fatty } \\
\text { acid }\end{array}$} & \multicolumn{6}{|c|}{ Incorporation at equilibrium ${ }^{a}(\%)$} \\
\hline & \multicolumn{3}{|c|}{ Transesterification } & \multicolumn{3}{|c|}{ Acidolysis } \\
\hline & \multicolumn{2}{|c|}{ Calculation $^{b}$} & \multirow[t]{2}{*}{ Found } & \multicolumn{2}{|c|}{ Calculation $^{b}$} & \multirow[t]{2}{*}{ Found } \\
\hline & Eqn. (1) & Eqn. (2) & & Eqn. (1) & Eqn. (2) & \\
\hline EPA & 31 & 26 & 33.0 & 32 & 26 & 33.5 \\
\hline DHA & 28 & 24 & 28.7 & 28 & 25 & 26.1 \\
\hline PA & 3 & 7 & 2.8 & 3 & 7 & 3.2 \\
\hline
\end{tabular}

${ }^{a}$ After $72 \mathrm{~h}$ reaction time. ${ }^{b}$ The calculations were based on the following initial fatty acid composition of (1) the cod liver oil substrate: 9.4\% EPA, 9.2\% DHA and 10.7\% PA; (2) the ethyl ester and free fatty acid concentrates of identical composition: $39.4 \%$ EPA, $35.2 \%$ DHA and $0 \%$ PA. Regarding the positional distribution of these fatty acids in the cod liver oil triglycerides, our results from the pancreatic lipase hydrolysis (see Table 2) were used in eqn. (2). 


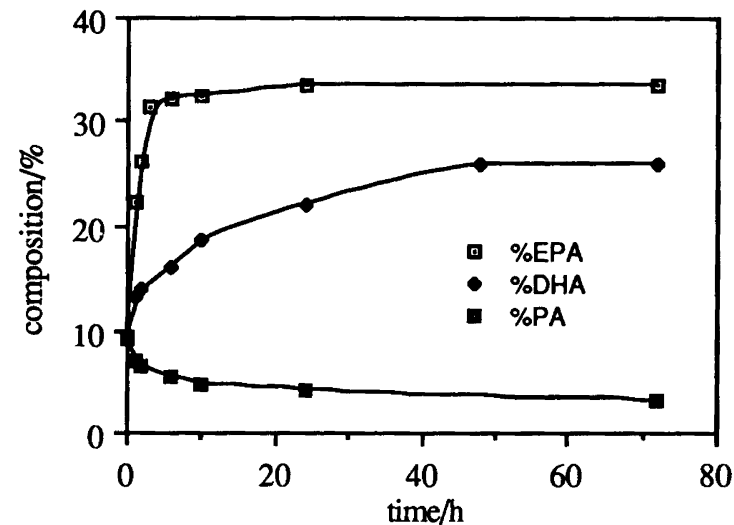

Fig. 3. Percentages of EPA, DHA and PA in triglycerides during acidolysis.

Hydrolysis side-reaction. It was evident from titration studies and iatroscan measurements that the hydrolysis sidereaction occurred to considerably lower extent in the acidolysis reaction compared with the transesterification. The extent of hydrolysis was found to be very much dependent on the moisture content of the lipase. ${ }^{17-19}$ With the $10 \%$ moisture content lipase an equilibrium involving 7-9\% free fatty acids was established within $10 \mathrm{~h}$ in the transesterification process. Similarly, iatroscan studies of the product from the acidolysis reaction indicated $4-5 \%$ extent of hydrolysis. The acidolysis process is apparently less prone to the partial hydrolysis because of the vast excess of free fatty acids present, the second product of such hydrolysis, shifting the equilibrium towards the triglycerides.

According to titration studies on ethyl esters and cod liver oil separately, ethyl esters were hydrolyzed at a faster rate but to a lesser extent than the triglycerides. Therefore, it is difficult to tell in which proportions ethyl esters and triglycerides of cod liver oil are hydrolyzed in a mixture. As a result of hydrolysis, detectable amounts of diglycerides were present in the equilibrium mixture according to TLC, whereas monoglycerides were not detected.

Comparison of the calculated and observed fatty acid compositions after interesterification. The results of fatty acid analyses and calculations of statistical composition are shown in Table 1. Random distribution seems to have been attained, considering the results in Table 1 . The measured composition of EPA and PA indicates that the assumption upon which eqn. (1) was based is apparently correct. DHA also seems to be randomly distributed after transesterification after $72 \mathrm{~h}$ but a significantly lower figure was obtained after acidolysis for $72 \mathrm{~h}$, the observed value being approximately midway between the two calculated results. In general, the acidolysis reaction is slower than transesterification, but it is first and foremost the slower DHA uptake that is the limiting factor regarding reaction time. It should, however, be stressed that in a number of previous experiments DHA incorporation levels very close to the calculated values according to eqn. (1) were obtained, and this supports our results that randomization takes place for both transesterification and acidolysis.

The possible explanations for the reduced rate of incorporation into triglycerides in the acidolysis reaction relative to transesterification are, firstly the large excess of free fatty acids in the system. Their effect on the hydrolysis equilibrium is to reduce the amount of diglycerides, the concentration of which might be critical to the rate of reaction. ${ }^{19}$ Secondly, there may be detrimental effects of free acids on the basic anionic support material of the lipase preparation. Blockages and inactivation may result, if acidic components bind electrostatically to the surface of the material. Lastly, the rate of uptake into the mid-position of glycerides is impeded. This would presumably be a result of the first explanation. Support for this is gained from Goderis et al. ${ }^{19}$ This group has found that large amounts of free fatty acids in an acidolysis reaction with immobilized lipase do indeed deter the hydrolysis of triglycerides and thus reduce the amount of diglycerides present. Furthermore, this group concluded that mono- and diglycerides reach equilibrium at any time during the reaction whereas the attainment of equilibrium between di- and tri-glycerides seemed to be rate-determining. This is consistent with the assumption that the concentration of intermediate diglycerides is critical to reaction rate.

Grignard reaction and pancreatic lipase catalyzed hydrolysis of cod liver oil. In order to measure the composition of the mid-position in cod liver oil, pancreatic lipase hydrolysis was performed and the resulting 2-monoglycerides were used as a measure of composition in the mid-position. Also, the Grignard reaction was run, 1,3- and 1,2(2,3)diglycerides isolated, and the necessary calculations performed indirectly to measure the composition of the midposition. The fatty acid composition of 1,3-diglycerides was taken as representative of the average composition in the end-positions, but the following equation was used to calculate the composition of the mid-position:

$\% X_{\mathrm{TG}_{-2}}=2 \% X_{\mathrm{DG}_{-1,2(2,3)}}-\% X_{\mathrm{DG}_{-1,3}}$

The methods of mid-position analysis were subsequently

Table 2. Comparison of mid-position analyses of cod liver oil, using pancreatic lipase catalyzed hydrolysis and the Grignard reaction.

\begin{tabular}{lllll}
\hline Position & & $\begin{array}{l}\text { Lipase } \\
\text { hydrolysis }\end{array}$ & $\begin{array}{l}\text { Grignard } \\
\text { reaction }\end{array}$ & $\begin{array}{l}\text { Literature }_{\text {values }^{8}} \\
\text { Position-2 }\end{array}$ \\
& $\%$ EPA & 12 & 14 & 12 \\
& $\%$ DHA & 16 & 14 & 20 \\
& $\%$ PA & 17 & 16 & 16 \\
Positions-1,3 & $\%$ EPA & 9 & 8 & 8 \\
& $\%$ DHA & 3 & 5 & 4 \\
& $\%$ PA & 9 & 9 & 11 \\
\hline
\end{tabular}




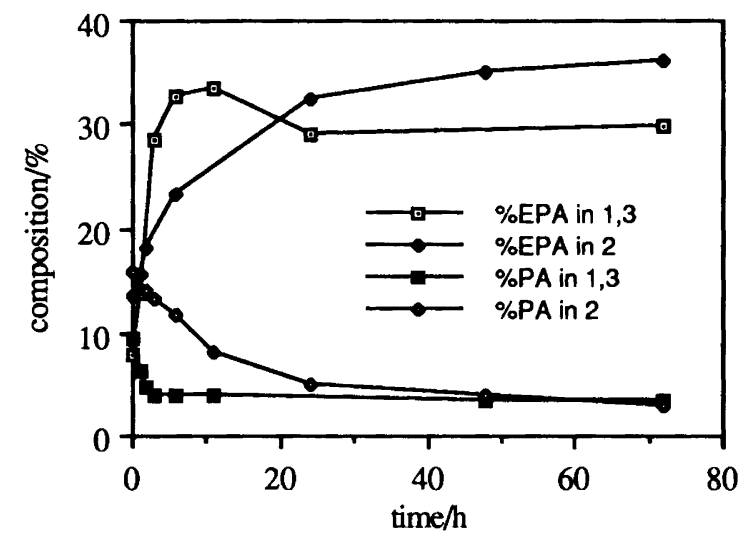

Fig. 4. Percentages of EPA and PA in end-positions and midposition during transesterification.

compared and Table 2 shows the results, together with the corresponding results for the end-positions (average). PA shows good correspondence, both between methods and with the literature. EPA is rather high according to the Grignard method but DHA appears anomalously low by that method. One should interpret the literature values cautiously, since seasonal variations, site of catch and other factors influence the $n-3$ fatty acid content markedly. ${ }^{20}$ Regarding the end-position analysis, there is a good agreement between methods for EPA and PA, but DHA again shows rather high degree of discrepancy.

Analysis of changes in the mid-position of triglycerides during interesterification. Originally, it was our intent to use pancreatic lipase catalyzed hydrolysis to investigate the composition of the mid-position, but when it was applied to $n-3$ enriched triglycerides previously made in our laboratory, hydrolysis proved extremely sluggish. Usually, the extent of hydrolysis did not exceed $20 \%$, whereas with cod liver oil levels of 40 and $50 \%$ hydrolysis were reached after 4 and $5 \mathrm{~min}$, respectively. This is in accord with earlier observations, which pertain to the fatty acid selectivity of

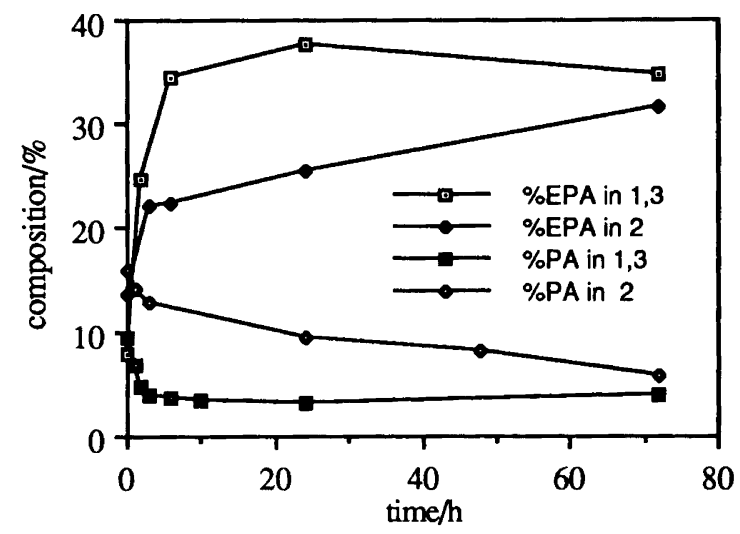

Fig. 5. Percentages of EPA and PA in end-positions and midposition during acidolysis. pancreatic lipase..$^{21}$ Therefore, the Grignard method,,$^{13}$ which was believed to display no such fatty acid selectivity, was used to monitor changes in the mid-position in the studies involving $n-3$ fatty acid concentrates.

The Grignard method was applied to the purified triglyceride fractions from interesterifications. 1,3 diglycerides were used as a measure of the mean composition in the end-positions but the mid-position was calculated using eqn. (3). EPA uptake and PA removal from positions 1,3 and 2 during transesterification are shown in Fig. 4. PA shows the most dramatic difference between end-positions and mid-positions but EPA also shows a difference. The greater difference in concentration of EPA in cod liver oil and the ethyl ester concentrate is probably responsible for the relatively faster incorporation of EPA into the midposition, compared with PA.

Similarly, purified triglycerides from acidolysis were subjected to mid-position analysis using the Grignard protocol. The results are shown in Fig. 5.

Comparing the curves, it is obvious that it takes a considerably longer reaction time for the acidolysis reaction to achieve random distribution of the fatty acids than observed for transesterification.

A dramatic difference is observed between the rates of change in the mid-position and the end-positions, indicating 1,3-specificity of the lipase during the acidolysis reaction, as was observed for transesterification. Factors other than lipase catalyzed interchange are presumably responsible for the changes in the mid-position, although lipase catalysis cannot be ruled out unequivocally.

Comparison of acidolysis and transesterification. In Fig. 6 the results from transesterification and acidolysis are compared further. It is immediately apparent that rates of change in 1,3 diglycerides and hence in the end-positions are comparable, but the mid-position seems to exclude PA at a much slower rate during acidolysis than during transesterification. Evidently, the changes in the mid-position are rate-determining for the attainment of equilibrium concentrations in the final triglyceride after the interesterification

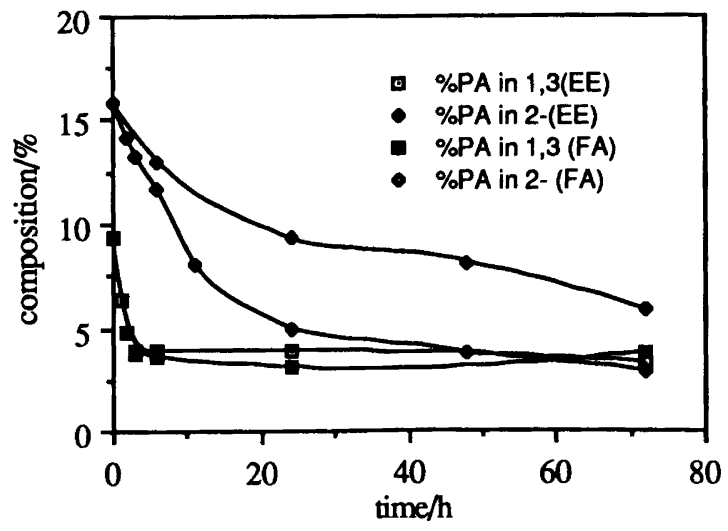

Fig. 6. Percentages of PA in end- and mid-positions during transesterification (EE) and acidolysis (FA). 
reactions. This effect of the mid-position is much more pronounced in the case of acidolysis. It is believed that the changes occurring in the mid-position do not result from lipase catalyzed reactions directly, since these changes take place over a long reaction time while the changes in the end-positions take place rapidly and 'equilibrate' after few hours of reaction. Schuch and Mukherjee ${ }^{22}$ have reported interesterifications with lipozyme in organic media to take place primarily at positions 1 and 3 , and since the group used a short reaction time of around $4 \mathrm{~h}$, significant changes in the mid-position were not detected. Considering this and the study of Goderis et al. (vide supra), it is apparent that interesterifications of the type in question with lipozyme occur through lipase catalyzed interchange in the end-positions, while rearrangements of intermediates such as diglycerides are responsible for changes in fatty acid composition of the mid-position of the triglycerides over extended reaction periods.

The peculiar behaviour of DHA (not shown) in the positional analyses warrants special comment. Consistently low values for DHA were found in 1,2(2,3)- and 1,3diglycerides after the Grignard reaction and subsequent TLC separation. This kind of behaviour has been observed before in our laboratory as well as in others ${ }^{23}$ and is possibly a consequence of the exposure of DHA to boric acid impregnated silica gel. However, the well established chemistry of the Grignard reaction does not seem to offer any explanation for the disappearance of this acid specifically, although there is a question as to whether the influence of the double bond one carbon-carbon bond closer to the carboxy group in DHA in increasing the reactivity of the ester can be ruled out entirely. The effect on DHA needs further study, but for the time being it is our belief that EPA and PA serve to demonstrate the 1,3-specificity of lipozyme during the interesterification processes.

\section{Conclusions}

During an interesterification process with lipozyme in organic media detectable amounts of lower glycerides are formed as a result of partial hydrolysis of fat with lipase. The glycerides have an opportunity to rearrange, possibly due to slightly 'protic' conditions (caused by free acid protons or residual water from lipase), subsequently reassembling with a new fatty acid moiety. These events are summarized in Fig. 7.

Our conclusions are further supported by randomization experiments with cod liver oil and lipase in the absence of a concentrate, which are not reported specifically in the present paper. In the presence of lipozyme, randomization of positional distribution occurred within 6 days under the same conditions as used in the interesterification reactions described. Furthermore, when acidolysis using fatty acids from cod liver oil with cod liver oil was performed, no change was observed in the fatty acid composition of the final triglycerides over a period of three days. This in-

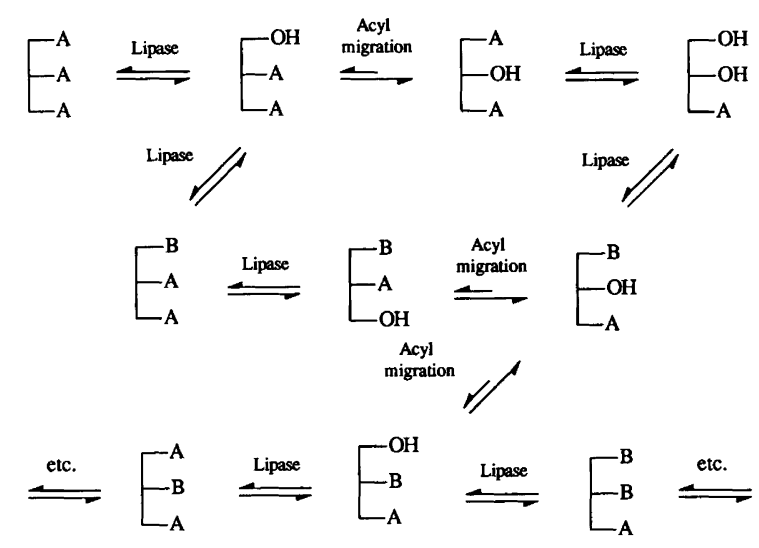

Fig. 7. Randomisation to diglycerides during interesterification in the presence of a lipase.

dicates that all fatty acid equivalents paticipate and the equation for calculating final composition of the triglycerides assuming random distribution of fatty acids is the one that applies.

The conclusion of this particular study is that lipozyme is 1,3-specific during transesterification and acidolysis reactions under solvent- and water-free conditions, thus adhering to its positional specificity as described for its action on emulsified substrates during hydrolysis reactions. ${ }^{24,25}$ Randomization of the original mid-position is a result of acyl migrations which are rather poorly understood as yet. Our belief is that these events are promoted by the lipase, which opens up the end-positions followed by the consequential non-lipase catalyzed migrations. Acyl migrations seem to be rate-determining for the attainment of a statistical equilibrium based on the random distribution of fatty acids in the glycerides in the systems that were studied.

Acknowledgements. The authors would like to thank the Icelandic University Research Fund for financial support, Dr. Hördur Kristjánsson at the Science Institute for valuable comments, Börkur Gunnarsson at the Icelandic Fisheries Laboratory for performing molecular distillations of ethyl esters and Tomas T. Hansen and Hanne Svanholm at Novo Nordisk, Bagsværd, Denmark, for kindly providing the enzyme preparation and performing iatroscan measurements.

\section{References}

1. Borgström, B. and Brockman, H. L., Eds., Lipases, Elsevier, Amsterdam 1984.

2. Haraldsson, G. G. In: Voigt, M. N. and Bhotta, R., Eds., Advances in Fisheries Technology and Biotechnology for Increased Profitability, Technomic Publishing Co., Inc., Pennsylvania 1990 , pp. 337-357.

3. Brockerhoff, B. and Jensen, R. G., Eds., Lipolytic Enzymes, Academic Press, New York 1974. 
4. Zaks, A. and Klibanov, A. M. Proc. Natl. Acad. Sci. USA 82 (1985) 3192.

5. Klibanov, A. Acc. Chem. Res. 23 (1990) 114.

6. Haraldsson, G. G., Höskuldsson, P. A., Sigurdsson, S. T., Thorsteinsson, F. and Gudbjarnason, S. Tetrahedron Lett. 30 (1989) 1671.

7. Bloomer, S. Triglyceride Interesterification by Lipases, Licentiate Thesis, Department of Biotechnology, University of Lund, Sweden 1990, pp. 54-62.

8. Brockerhoff, H., Hoyle, R. J., Hwang, P. C. and Litchfield, C. Lipids 3 (1967) 24.

9. Haraldsson, G. G., Höskuldsson, P. A., Gunnarsson, O. and Gunnarsson, B. Unpublished results.

10. Gilman, H. and Cartledge, F. K. J. Organomet. Chem. 2 (1964) 447.

11. Fine, J. B. and Sprecher, H. J. Lipid Res. 23 (1982) 660

12. Hamilton, R. J. In: Hamilton, R. J. and Rossell, J. B., Eds., Analysis of Oils and Fats, Elsevier, New York 1986, pp. 265267.

13. Brockerhoff, H. J. Lipid Res. 8 (1967) 167.

14. Mattson, F. H. and Volpenhein, R. A. J. Lipid Res. 2 (1961) 58.

15. Einig, R. G. and Ackman, R. G. J. Am. Oil Chem. Soc. 64 (1987) 499.
16. Haraldsson, G. G. In: Shukla, V. K. S. and Hölmer, G., Eds., Proceedings of the 15th Scandinavian Symposium on Lipids, Lipidforum 1989, pp. 245-248.

17. Dordick, J. S. Enzyme Microbiol. Technol. 11 (1989) 194.

18. Yamane, T. In: Applewhite, T. H., Ed., World Conference on Biotechnology for the Fats and Oils Industry, American Oil Chemists' Society, Illinois 1988, pp. 17-22.

19. Goderis, H. L., Ampe, G., Feyten, M. P., Fouwé, B. L., Guffens, W. M., Van Cauwenbergh, S. M. and Tobback, P. P. Biotechnol. Bioeng. 30 (1987) 258.

20. Stansby, M. E. J. Am. Oil Chem. Soc. 56 (1981) 13.

21. Brockerhoff, H. Biochim. Biophys. Acta 212 (1970) 92.

22. Schuch, R. and Mukherjee, K. D. In: Applewhite, T. H., Ed., World Conference on Biotechnology for the Fats and Oils Industry, American Oil Chemists' Society, Illinois 1988, pp. 328-329.

23. Brockerhoff, H., Hoyle, R. J. and Huang, P. C. Can. J. Biochem. 44 (1966) 1519.

24. Huge-Jensen, B., Galluzo, D. R. and Jensen, R. G. Lipids 22 (1987) 559.

25. Huge-Jensen, B., Galluzo, D. R. and Jensen, R. G. J. Am. Oil Chem. Soc. 65 (1988) 905.

Received December 5, 1990. 